\title{
Comprensión y resolución de problemas matemáticos desde la macroestructura del texto
}

Leidi Viviana Montero Yas $^{1}{ }^{[}$, Jair Andres Mahecha Farfán

Universidad Arturo Prat - Chile

\section{Resumen}

Una preocupación de los docentes de básica primaria en Colombia es el bajo desempeño de los estudiantes en el área de matemáticas y en las pruebas de Estado. A pesar de que existe aptitud en la solución de algoritmos, hay dificultad en la comprensión de los enunciados de los problemas matemáticos. El propósito de este artículo es compartir una propuesta metodológica que contribuya al mejoramiento de los niveles de comprensión y resolución de problemas matemáticos, desde el concepto de macroestructura textual, al integrar las áreas de matemáticas y lenguaje. La investigación, de carácter cualitativo, se desarrolló en el marco de la investigación acción. Se hizo una fundamentación teórica. Hubo tres fases claves: el diagnóstico, el análisis de la información $y$, con base en estos dos, la elaboración de la propuesta metodológica. Se presentan como resultados: el diagnóstico de las dificultades y fortalezas al resolver problemas matemáticos, el diseño de la propuesta metodológica con la reconstrucción de macroestructuras en el análisis de enunciados, y el principio de interdependencia entre matemáticas y lenguaje.

Palabras clave: resolución de problemas, comprensión, matemáticas, lingüística 


\title{
Understanding and solving mathematical problems from the macro-structure of the text
}

\begin{abstract}
A concern of primary school teachers in Colombia is the low performance of students in mathematics and in State tests. Despite students are capable of solving algorithms, it is difficult for them to understand the statements of mathematical problems. The purpose of this article is to share a methodological proposal that contributes to the improvement of the levels of understanding and solving mathematical problems, from the concept of textual macro-structure, by integrating mathematics and language. The research, with a qualitative approach, was developed within the framework of action research. A theoretical foundation was made. There were three key phases: the diagnosis, the analysis of the information and, based on these two, the elaboration of the methodological proposal. The results are the diagnosis of difficulties and strengths when solving mathematical problems, the design of the methodological proposal with the reconstruction of macro-structures in the analysis of statements, and the principle of interdependence between mathematics and language.
\end{abstract}

Key words: problem solving, comprehension, mathematics, linguistics

\section{Compreensão e resolução dos problemas matemáticos a partir da macroestrutura do texto}

\section{Resumo}

Uma preocupação dos professores do ensino da primaria na Colômbia é o fraco desempenho dos alunos na área de matemáticas e nas provas do Estado. Apesar de que existe aptidão na solução de algoritmos, há dificuldade na compreensão dos anunciados dos problemas matemáticos. O proposito deste artigo é compartilhar uma proposta metodológica que contribuía para a melhoria dos níveis de compreensão e resolução dos problemas matemáticos, a partir do conceito de macroestrutura textual, ao integrar as áreas de matemática e linguagem. A pesquisa, de caráter qualitativo, foi realizada no âmbito da pesquisa ação. Fez-se uma fundamentação teórica. Houve três fases principais: o diagnóstico, a análise das informações e, com base nesses dois, a elaboração da proposta metodológica. Apresentam-se como resultados: o diagnóstico das dificuldades e fortalezas na solução de problemas matemáticos, o desenho da proposta metodológica com a reconstrução de macroestruturas na análise de enunciados, e o princípio da interdependência entre matemáticas e a linguagem.

Palavras-chave: resolução de problemas, compreensão, matemática, linguística

\section{Introducción}

Este trabajo es un aporte para la integración curricular entre el área de matemáticas y lenguaje. También es una contribución para la reducción de distancias entre dichas áreas, puesto que el análisis de enunciados de los problemas matemáticos y la comprensión de 
textos son procesos interdependientes que no deben ser fraccionados, sino integrados. Esta investigación parte de una situación predominante en las clases de matemáticas, donde se presenta una diferencia significativa entre el número de aciertos de los estudiantes al desarrollar algoritmos y el número de aciertos al resolver problemas que involucran algoritmos similares. La menor cantidad de aciertos se debe a las dificultades al comprender los enunciados de los problemas. Al respecto, Pérez, Hernández y Álvarez (2015) manifiestan que "en la práctica educativa se aprecia un insuficiente nivel de comprensión en el desempeño de los escolares" (p. 2).

Con base en lo anterior, se formula el problema de investigación: ¿Cómo mejorar la comprensión y resolución de problemas matemáticos, desde el concepto de macroestructura textual, en estudiantes de grado quinto de primaria? Se involucran así dos procesos claves en el desarrollo académico de estos estudiantes: los problemas de estructura multiplicativa, que son propios de ese nivel escolar, y la construcción de macroestructuras dentro del proceso de comprensión lectora (Herrada \& Herrada, 2017; Montes, Rangel \& Reyes, 2014) como uno de los conceptos contemporáneos esenciales en la comprensión de lectura desde la lingüística del texto.

Todo proceso mental —como la resolución de problemas y la comprensión- requiere de etapas de desarrollo y fortalecimiento (Rodríguez, Gregori, Riveros \& Aceituno, 2017; Schoenfeld, 1994). Aunque dichas etapas se dan de manera individual, es deber de la escuela contribuir con actividades, estrategias y herramientas para que los estudiantes desarrollen cierta habilidad. Para atender esta situación, se planteó como objetivo principal el diseño de una propuesta metodológica que contribuyera al mejoramiento de los niveles de comprensión y resolución de problemas aritméticos de estructura multiplicativa, desde el concepto de macroestructura textual. Así mismo, como metas específicas se propuso: el desarrollo de un diagnóstico para identificar dificultades y fortalezas específicas en la comprensión de enunciados; la apropiación de herramientas y desarrollo de habilidades inferenciales de lectura desde el concepto de macroestructura; y la aplicación de estas en el análisis de los enunciados de los problemas.

Para responder al cómo de la investigación, se hizo una revisión de la fundamentación teórica centrada en las teorías y conceptos relevantes. El proceso metodológico se diseñó según la investigación acción. Se inició con la estructuración de la matriz categorial y el diseño de instrumentos. Luego se llevó a cabo la intervención con la población. Por último, y en lo que respecta al producto de la investigación, se presentó el análisis de datos de los resultados obtenidos.

\section{Perspectiva de la educación matemática en Colombia}

El cambio de paradigmas en la enseñanza de las matemáticas no es tarea sencilla. En algunas instituciones educativas aún se "enseña matemáticas" como un proceso basado, en gran parte, en el aprendizaje de algoritmos. "Esto es especialmente interesante debido a la presencia de contenidos en el currículo que se prestan claramente al diseño de algoritmos y a una experimentación mayor que la que permite la enseñanza tradicional" (Briz \& Serrano, 2018, p. 133). Aunque la habilidad para la mecanización y repetición de procedimientos se convierte en indicador de óptimo desempeño, esto no muestra el nivel de comprensión. Así, las matemáticas se convierten en un campo aislado, desligado de otras áreas, en donde 
muchos estudiantes muestran dificultad para comprender su aplicabilidad o funcionalidad en el entorno y presentan bajo desempeño no solo a nivel institucional, sino también en las pruebas externas, como lo presenta el Instituto Colombiano para el Fomento de la Educación Superior, ICFES. A esto se agrega cierta influencia cultural en la que se transmite predisposición acerca de su difícil aprendizaje.

Murcia y Henao (2015), en su mirada sobre la enseñanza de las matemáticas, ratifican que "la dificultad radica en que los contenidos y las competencias que se quieren desarrollar se vuelven independientes, sin el concurso transversal de otros saberes" (p. 27). A ello agregan que

los bajos niveles de lectura y desde luego, comprensión de lectura de los estudiantes y la cultura del facilismo y posibles errores en los procedimientos para la promoción escolar y la ausencia de acompañamiento en casa, necesariamente llevan al fracaso escolar. (p. 27)

El Ministerio de Educación Nacional [MEN] (1998) plantea como propósito de esta área desarrollar el pensamiento matemático con miras a ayudar a las personas a dar sentido al mundo que les rodea; a comprender los significados que otros construyen o cultivan; y a desarrollar y apropiarse de una serie de instrumentos que les permiten comprender la realidad y actuar en ella. Uno de los procesos generales en que tienen más dificultad los estudiantes es el planteamiento y resolución de problemas, dado que se enfrentan a una situación ya sea verbal o escrita en la que requieren varios procesos mentales como la comprensión, el análisis, la producción y la comunicación, solo por mencionar algunos. Para ellos es más sencillo repetir procedimientos que comprender su sentido.

\section{Problemas matemáticos}

En términos generales, los problemas se pueden comprender como todas aquellas situaciones que se le presentan a un sujeto y que requieren de una solución por medio del uso de un conjunto de procesos mentales. Estos se enuncian en palabras, ya sea oralmente o en forma escrita. De igual forma, desde el campo de la lingüística, cuando se habla de situación, se hace referencia a la relación mundo-tiempo, en un mundo posible. Mundo posible se entiende como una serie de proposiciones o ideas de un hecho posible, en otras palabras, cada situación es un conjunto de hechos particulares (Van Dijk, 1980).

Estos mundos creados desde el entorno inmediato pueden tener una incógnita, un dato desconocido o asunto por resolver; elementos que permiten la concreción de una situación problema. Si la solución requiere de procesos mentales y conocimientos básicos del pensamiento matemático, se convierte en un problema matemático. Los problemas matemáticos no cuentan con una definición única. Diversos autores establecen caracterizaciones que permiten describirlos y tipificarlos. Blanco (2015) hace una compilación y análisis de las diferentes posturas de autores como Carrillo (1995), House, Wallace y Johnson (1983) y Pino (2013), entre otros; análisis en el que sintetiza:

Un problema es una situación en la que se formula una tarea que debe ser desarrollada, y en la que, en un ambiente de discusión, de incertidumbre y de comunicación, se pretende alcanzar unos objetivos. En este propósito cuantitativo o no, pero que debe requerir técnicas matemáticas, el proceso a seguir no debe ser conocido inmediata y 
fácilmente. Se requiere en todo caso una voluntad de atacar el problema provocado, por la necesidad de la solución o bien por algún tipo de motivación. (Blanco, 2015, p. 84)

Desde esta mirada, cabe decir que todo problema requiere un proceso para hallar su solución (Castro, 2017). Para ello es necesario conocer los elementos que lo constituyen y sus características. Ormrod (2005) afirma que cualquier tipo de problema tiene por lo menos tres elementos constituyentes: "los datos o información que se suministra, el objetivo que corresponde el estado final al que se pretende llegar y las operaciones o acciones que es posible realizar para lograr o acercarse al objetivo" (p. 417). Pese a esta contextualización de los problemas matemáticos, en muchas prácticas educativas este proceso se reduce a la práctica operatoria - mecanización de operaciones básicas- como un fin, lo cual podría denominarse como un ejercicio rutinario de mecanización (Mora, 2003). No obstante, para el sistema educativo nacional los problemas matemáticos van más allá de ser un simple ejercicio mecánico. El MEN (1998) plantea que en el espacio de resolución se deben promover procesos que desarrollen un aprendizaje innovador y que permitan estimular la creatividad, la originalidad y el razonamiento matemático.

\section{Resolución de problemas matemáticos}

Así como no se puede hablar de una clasificación específica de los problemas matemáticos, tampoco existe una ley única y efectiva para la resolución de problemas (Leal \& Bong, 2015). Por ello se encuentra amplia bibliografía de autores que plantean estrategias que al ser aplicadas contribuyen a un óptimo desarrollo del proceso mencionado y que además rompen con el esquema tradicional de resolución utilizado en básica primaria, que consiste en dividir la hoja del cuaderno en dos columnas, una para el análisis y otra para la operación, lo cual limita la creatividad del estudiante. Los principales aportes al respecto se sustentan desde las teorías de Polya (1965, citado por Ormrod, 2005), quien confirma que no existe un conjunto de reglas o axiomas para la resolución; que todos los seres humanos poseen estilos cognitivos, ritmos de aprendizaje diferentes; y que hay algunos de ellos con más habilidad para resolverlos que otros de su misma edad. Estos sujetos pueden aplicar - muchas veces sin darse cuenta - toda una serie de técnicas y métodos que resultan adecuados y eficientes para afrontar los problemas. Para este autor, en la resolución de un problema prima la reflexión. Hasta se pueden utilizar pasos o estrategias que no se habían ensayado antes para dar la respuesta. Dicha característica es lo que distingue un problema de un ejercicio. Para el proceso de resolución, Polya (1965) propone cuatro etapas esenciales: comprender el problema, trazar un plan para resolverlo, poner en práctica el plan y comprobar resultados.

Desde una perspectiva más reciente, Blanco y Caballero (2015) proponen un modelo integrado de resolución de problemas, que considera tanto los aspectos cognitivos como los afectivos, estructurado en cinco fases como lo presenta la figura 1.

Cada fase tiene un objetivo específico: tomar conciencia y gestionar sus respuestas cognitivas, seleccionar posibles estrategias que conduzcan a la solución, ejecutar la selección anterior, revisar respuestas y, por último, reflexionar sobre la tarea realizada; esto sin perder de vista el propósito general, que es ayudar a los estudiantes a aprender a resolver problemas a partir de su propio estilo (Blanco \& Caballero, 2015). 


\begin{tabular}{|c|c|c|}
\hline & HEURÍSTICOS & CONTROL EMOCIONAL \\
\hline $\begin{array}{l}\text { 1. Análisis/comprensión y } \\
\text { familiarización con el } \\
\text { enunciado }\end{array}$ & $\begin{array}{l}\text { Releer el enunciado, notaciones, } \\
\text { gráficos, extracción de datos explícitos } \\
\text { e implícitos, de objetivos, determinar } \\
\text { contextos y condiciones... }\end{array}$ & \multirow{3}{*}{$\begin{array}{l}\text { Respiración } \\
\text { Relajación muscular } \\
\text { Autoinstrucciones }\end{array}$} \\
\hline $\begin{array}{l}\text { 2. Búsqueda de estrategia/s de } \\
\text { solución }\end{array}$ & $\begin{array}{l}\text { Relacionar datos-incógnitas, } \\
\text { conocimientos previos, re-enunciar el } \\
\text { problema, enunciar subproblemas... }\end{array}$ & \\
\hline $\begin{array}{l}\text { 3. Ejecución de la/s } \\
\text { estrategia/s }\end{array}$ & $\begin{array}{l}\text { Registrar y explicar todos los pasos, } \\
\text { actuar con rigor, orden y precisión... }\end{array}$ & \\
\hline $\begin{array}{l}\text { 4. Examen/control de la } \\
\text { solución y del proceso }\end{array}$ & $\begin{array}{l}\text { Analizar la consistencia de la solución y } \\
\text { del proceso, resolver de modo diferente, } \\
\text { transferencia y generalización... }\end{array}$ & Autoinstrucciones \\
\hline $\begin{array}{l}\text { 5. ¿Cómo me siento? } \\
\text { ¿Qué he aprendido? }\end{array}$ & $\begin{array}{l}\text { Valora tu actitud, tus avances y } \\
\text { proposición de una pequeńa meta }\end{array}$ & $\begin{array}{c}\text { Autoinstrucciones } \\
\text { Reflexiones para modificar } \\
\text { su afectividad }\end{array}$ \\
\hline
\end{tabular}

Figura 1. Método de resolución de problemas (Blanco \& Caballero, 2015, p. 113).

\section{Complejidad de la resolución de problemas por falta de comprensión}

Desde los aportes de Polya (1965) hasta los más recientes, o influyentes, como los de Blanco (2015), se coincide en que la primera fase de resolución de problemas matemáticos se centra en su comprensión, sin la cual las etapas posteriores quedan sin fundamento, es decir, sin entender el problema, avanzar en él es inoficioso. Por ello se debe centrar la mirada en los procesos de comprensión de los enunciados. Frente a esta relación entre lenguaje y resolución de problemas, Cárdenas y Blanco (2015) enfatizan en que dicha relación se concreta en diferentes momentos relacionados de forma global, pero sobre todo en los espacios en los que el niño "expresa oralmente y por escrito el significado de los datos, la situación planteada, el proceso seguido y las soluciones obtenidas" (p. 33). Así, el lenguaje se convierte en elemento constitutivo en el proceso de resolución. Al respecto, se debe tener en cuenta que

la comunicación es un medio necesario para que se produzca aprendizaje. La expresión oral o escrita del trabajo realizado obliga a un esfuerzo de síntesis y precisión para que el interlocutor nos entienda, lo que nos ayuda a profundizar en la comprensión de lo realizado. (Cárdenas \& Blanco, 2015, p. 33)

En este orden de ideas, Puig y Cerdan (1995) ahondan en las características de los problemas matemáticos y mencionan características generales en cuanto a la estructura de los enunciados de cada situación. Plantean que estos tienen tres componentes comunes y definitivos en su comprensión: el componente sintáctico - que se refiere a la estructura superficial-, la estructura lógica de acuerdo con la clase y, finalmente, el componente semántico - que se refiere al sentido entre proposiciones-; lo cual evidencian la relación directa entre la resolución de problemas matemáticos con la lingüística.

\section{Comprensión}

El proceso mental denominado comprensión hace referencia a ser capaz de tomar el 
conocimiento y utilizarlo en diferentes formas y contextos. En palabras de Perkins (1995), "la comprensión no puede ser vista como un estado de posesión, sino como un estado de capacitación, ya que implica no solo el tener el conocimiento o la información, sino la habilidad de hacer ciertas cosas con él” (p. 82). Es por esto que se puede catalogar la relación recíproca entre tener conocimiento y su respectivo uso o aplicabilidad como propiedad fundamental de la comprensión (Pérez \& Hernández, 2014). Sin embargo, en diferentes contextos, como el educativo, se tiende a confundir este término con competencia, a tal punto que se plantean como sinónimos. Para evidenciar que se ha desarrollado un proceso de comprensión, Perkins (1995) plantea que existen procesos y acciones específicas, a los que denomina actividades de comprensión, entre las que se encuentran: la explicación, ejemplificación, aplicación, justificación, comparación y contraste, contextualización y generalización, entre otras. Por lo anterior, se puede afirmar que la comprensión es entonces un proceso mental abarcador de otros procesos. Así mismo, todos los sujetos están en continuo desarrollo de comprensiones. Sin embargo, en los procesos de enseñanzaaprendizaje, cada disciplina o campo de conocimiento estudia y plantea cómo se deben desarrollar dichos procesos desde las características propias de la disciplina.

En el campo de la lingüística, comprender es la asignación de significado, el cual facilita el procesamiento de la información. Para que se produzca dicho proceso, se requieren ciertas etapas, descritas por Van Dijk (1980) en su análisis del discurso. Partiendo de este planteamiento, Pérez (2006) se refiere a la comprensión de lectura como "el proceso cognoscitivo por el cual se reconstruye en la mente del lector la información transmitida por el autor del texto a través de un canal" (p. 75). De igual forma, para Zubiría (1995), no es conveniente desconocer que uno de los prerrequisitos de la lectura es la producción intelectual del texto realizada por el autor. Por consiguiente, la tarea del lector está orientada hacia el desciframiento, la inferencia y la reconstrucción.

Los textos y sus niveles - macroestructuras y microestructuras-

Para Bernárdez (1982), el texto, además de ser la unidad fundamental de comunicación, debe cumplir con ciertas características básicas para reconocerlo como: la unidad —cuando permite percibirlo como un todo-; el sentido semántico y sintáctico, que hace referencia a la coherencia - relaciones lógicas entre proposiciones - y la cohesión —conexión lógica de las relaciones_; la intencionalidad — aunque no sea explícita, se infiere-; y por último, todo texto debe tener un cierre - en ocasiones informal- que permite la reconstrucción global. Los enunciados de los problemas matemáticos, al cumplir con estos elementos, desde la lingüística también son reconocidos como textos.

Todo texto presenta dos niveles en cuanto a su estructura, ya sea local - micro-o global - macro- Van Dijk (1980) utiliza el término microestructura para denotar la estructura local de un discurso, es decir, la estructura de las oraciones y las relaciones de coherencia y conexión entre ellas. Mientras que la macroestructura, a diferencia de la microestructura, no se hace, sino que se reconstruye; en otras palabras, con base en un conjunto de proposiciones menores, se construye una mayor que las contenga a todas; es decir, organiza el sentido global del texto. Van Dijk introduce este término para dar cuenta del contenido global de un discurso.

La construcción de macroestructuras da cuenta de la comprensión 
Para efectos del trabajo investigativo, se tuvo en cuenta lo propuesto por Van Dijk (1980, citado por Serna \& Díaz, 2014), quien afirma que una de las primeras etapas en la comprensión textual es la supresión; es decir, la organización y reducción de grandes cantidades de información compleja: esto se produce cuando el conjunto de palabras $\mathrm{u}$ oraciones se interpretan en proposiciones - entendiendo la proposición como una unidad semántica-, las cuales se encadenan formando lo que se conoce como hecho cognoscitivo. En segundo lugar, él sugiere que hay que establecer las relaciones condicionales entre las proposiciones o hechos. Se refiere específicamente a la coherencia y cohesión semántica entre la información organizada y reducida - en ocasiones los enlaces también se emiten desde la memoria o hechos de la interacción social-. Este proceso conlleva a la construcción de una macroproposición, es decir, a la extracción de las proposiciones esenciales que muestra su respectiva relación. Cuando las macroproposiciones se encadenan y mantienen nexos entre ellas, se forma una estructura semántica —etapa denominada generalización-.

Este proceso de reconstrucción -macroestructura- se puede analizar cómo proceso inverso a la construcción - microestructura-, dado que se trata básicamente de llegar a la idea general de la que se partió en el momento de construir el texto. De igual forma trae consigo unas etapas de desarrollo, a las que Van Dijk (1980) define como macrorreglas:

Supresión: dada una secuencia de proposiciones, se suprimen todas las que no sean presuposiciones de las proposiciones subsiguientes de la secuencia.

Generalización: Dada una secuencia de proposiciones, se hace una proposición que contenga un concepto derivado de los conceptos de la secuencia de proposiciones, y la proposición así construida sustituye a la secuencia original.

Construcción: Dada una secuencia de proposiciones, se hace una proposición que denote el mismo hecho denotado por la totalidad de la secuencia de proposiciones, y se sustituye la secuencia original por la nueva proposición. (p. 48)

\section{Metodología}

La investigación tuvo un carácter cualitativo, orientado desde la investigación-acción diagnóstica. El proceso requirió de la identificación de: el grado de dificultad de los estudiantes en el proceso de resolución de este tipo de problemas; sus fortalezas y debilidades en cuanto a la interpretación y manejo de la información dada en el enunciado; el desarrollo de una serie de acciones, como el desarrollo del concepto de macroestructura textual en el análisis de textos cortos para fortalecer los niveles de comprensión de lectura; y su aplicación en la comprensión y resolución de dichos problemas para así mismo generar la propuesta. Dentro de la tipología de investigación-acción del modelo lewiniano, mencionado por Arnal, Rincón y Latorre (1992), en la investigación-acción diagnóstica "el investigador recoge los datos, establece un diagnóstico y recomienda unas medidas de acción” (p. 250).

La población seleccionada pertenecía a la localidad séptima de Bogotá - Bosa-, barrio El Recreo. Estuvo conformada por 43 estudiantes, 24 niñas y 19 niños con edades que oscilan entre los 9 y 12 años, pertenecientes al grado 503 de la Institución Educativa Distrital Leonardo Posada Pedraza. La muestra de esta investigación corresponde a la misma población, debido a que el número de participantes es suficiente para desarrollar el trabajo diseñado. 
Los instrumentos que se utilizaron se describen a continuación:

Pruebas de diagnóstico.

Para determinar el estado inicial de la población, en cuanto a las dificultades en el proceso de comprensión y resolución de problemas matemáticos de estructura multiplicativa, se desarrollaron tres fases diagnósticas. La primera consistió en una actividad en clase, que pretendió contrastar los procesos de solución de algoritmos cuando se presentan como ejercicios de aplicación, con aquellos que están inmersos en un problema o situación matemática, de tal forma que permitieran identificar si la dificultad radica en el desarrollo del algoritmo o en el proceso de comprensión de situaciones que requieren del mismo.

La segunda fase fue una prueba más detallada que permitía conocer el análisis y tratamiento que daba el estudiante a la información proporcionada en el enunciado de un problema matemático de estructura multiplicativa, así como su nivel de comprensión de lectura de este, con el fin de identificar sus fortalezas y debilidades en dicho análisis.

Finalmente, la tercera fue una prueba elemental de comprensión de lectura, cuyo propósito se centró en la identificación del nivel de comprensión - literal o inferencialque demuestra el estudiante en el análisis de una historia corta.

Las fases diagnósticas y sus respectivos criterios de análisis se estructuran en el siguiente esquema:

Tabla 1

Fases diagnósticas y elementos de análisis

\begin{tabular}{|c|c|c|}
\hline Fase & Criterio de análisis & Indicador \\
\hline $\begin{array}{l}\text { Actividad en clase de } \\
\text { algoritmos y problemas }\end{array}$ & $\begin{array}{l}\text { Desarrollo de algoritmos } \\
\text { Solución de problemas que } \\
\text { requieren de un algoritmo } \\
\text { para su desarrollo }\end{array}$ & $\begin{array}{l}\text { Resuelve correctamente los algoritmos de multiplicación } \\
\text { teniendo en cuenta las reglas y relaciones que implica. } \\
\text { Resuelve correctamente los algoritmos de división } \\
\text { teniendo en cuenta las reglas y relaciones que implica. } \\
\text { Identifica el algoritmo que se ajusta o requiere cada } \\
\text { situación o problema presentado y lo aplica en la forma } \\
\text { apropiada para hallar la solución al problema. }\end{array}$ \\
\hline $\begin{array}{l}\text { Prueba específica análi- } \\
\text { sis de problemas }\end{array}$ & $\begin{array}{l}\text { Identificación de informa- } \\
\text { ción del enunciado } \\
\text { Organización y relación de } \\
\text { la información } \\
\text { Desarrollo de procedimien- } \\
\text { tos acordes }\end{array}$ & $\begin{array}{l}\text { Reconoce las proposiciones que indican datos cono- } \\
\text { cidos e identifica valores o cantidades necesarios para } \\
\text { encontrar la solución del problema. } \\
\text { Reconoce la o las proposiciones que indican datos des- } \\
\text { conocidos, e identifica cuál es el valor, cantidad o in- } \\
\text { cógnita que se debe averiguar en el proceso de solución. } \\
\text { Reconoce la información o datos poco relevantes que se } \\
\text { pueden omitir en el proceso de solución del problema. } \\
\text { Establece la correspondencia adecuada entre las canti- } \\
\text { dades y las medidas para encontrar el posible algoritmo } \\
\text { que determina el dato desconocido. } \\
\text { Desarrolla el algoritmo en forma correcta, teniendo en } \\
\text { cuenta las reglas y relaciones que requiere. }\end{array}$ \\
\hline
\end{tabular}




\begin{tabular}{|l|l|l|}
\hline $\begin{array}{l}\text { Prueba elemental de } \\
\text { comprensión de lectura. }\end{array}$ & $\begin{array}{l}\text { Extrae la información dada en el texto, es decir lo que } \\
\text { dice de manera explícita o comprensión del significado } \\
\text { local de sus componentes. }\end{array}$ \\
Lectura inferencial & $\begin{array}{l}\text { Interpreta o descubre la información implícita que hay } \\
\text { en el texto, es decir reconoce las intenciones comuni- } \\
\text { cativas o una comprensión global del contenido y los } \\
\text { saberes. }\end{array}$ \\
\hline
\end{tabular}

Fuente: elaboración propia

La estructura de la actividad en clase, correspondiente a la fase 1 -algoritmos y problemas-, estuvo compuesta por 14 ejercicios, que a su vez se dividieron en dos partes. La primera presentó siete algoritmos de multiplicación y/o división básicos para estudiantes de este grado de escolaridad, mientras que la segunda presentó siete problemas matemáticos de estructura multiplicativa cuya solución se podía hallar con el desarrollo de algoritmos similares a los planteados en la primera parte.

En la fase 2 se presentó una prueba más específica, con cinco problemas matemáticos de estructura multiplicativa - tanto de isomorfismo de las medidas como de producto de medidas-. Durante el proceso de resolución de cada uno de los problemas, el estudiante debía además utilizar y/o desarrollar un algoritmo para solucionar la situación. Debía establecer los criterios bajo los cuales lo seleccionó, identificar datos o valores conocidos, datos o valores desconocidos y la información poco relevante, y verificar si la respuesta satisfacía la pregunta del problema.

La última prueba presentaba una lectura de una historia corta, que tenía cinco preguntas de comprensión - literales e inferenciales-, en la que los estudiantes debían mostrar su habilidad para la interpretación local y global de dicha historia.

\section{Observación libre.}

Debido a la forma de este tipo de investigación, los diálogos directos con la población fueron instrumentos fundamentales para la recolección de información, así como las preguntas dentro de los espacios de socialización, donde se observaba directamente el trabajo y las características del estudiante en las sesiones de trabajo.

\section{Observación estructurada.}

Al igual que la observación libre, el propósito es la recolección de información desde la observación de la población, solo que en este tipo de observación se tiene en cuenta una estructura previa. Para efectos del presente trabajo, se tuvo en cuenta el desarrollo de las pruebas diagnósticas y las actividades planteadas en los talleres iniciales diseñados.

\section{Análisis de resultados}

\section{Diagnóstico.}

Frente al análisis y selección de información en los enunciados de los problemas matemáticos de estructura multiplicativa, se amplió la observación hacia la clasificación y 
correspondencia que los estudiantes realizaban a los datos de los enunciados antes mencionados. Durante las sesiones de trabajo y el desarrollo de las actividades propuestas se encontró que:

- La mayoría de los estudiantes seleccionó datos numéricos como parte del problema, sin tener en cuenta a qué o a cuál categoría de datos pertenecía, es decir, que en los casos en los cuales el problema presentaba dos categorías de datos, como por ejemplo paquetes y yogures, los datos no se clasificaron de acuerdo con su categoría, sino que fueron puestos como términos del algoritmo seleccionado. Incluso datos numéricos que no correspondían a ninguna categoría, los distractores, también fueron tenidos en cuenta en el momento de plantear el algoritmo.

- En los enunciados que presentaron una de las medidas en forma no numérica -es decir, con el uso de términos como "un", "una", "diariamente", "cada", etc.-, los estudiantes no los identificaron como una medida. Por lo tanto, tampoco los clasificaron en una categoría o tipo de medida. Solo los reconocieron como parte del texto del enunciado.

- Muy pocos estudiantes establecieron relaciones de correspondencia entre los datos, teniendo en cuenta sus categorías y medidas. Algunos lo hicieron a través de dibujos, otros de esquemas, pero la tendencia fue a omitir las relaciones que se dieron entre estas y reducirlas a los términos de un algoritmo seleccionado, por la correspondencia con el tema de la clase o por la poca comprensión de la situación, como ya se describió en la fase diagnóstica.

- Debido a la dificultad para establecer las relaciones de correspondencia entre las medidas y sus categorías dentro de un problema de estructura multiplicativa, no se identificó el operador - ya fuera escalar o función- que le permitía hallar la respuesta a la situación.

- Los estudiantes no conocían ni utilizaban el procedimiento de regla de tres, por esto las situaciones que requerían de su uso no fueron solucionadas.

- Esta población presentó mayor aptitud para organizar la información y las medidas de un problema como una relación de producto; es decir si se conocían dos medidas, se buscaba una tercera como producto o resultado de una multiplicación. En los casos donde se conocía el producto y una medida, se buscaba otra medida utilizando el proceso inverso, la división.

En el proceso de resolución de problemas de estructura multiplicativa, en cuanto a la interpretación y manejo de la información dada en el enunciado - luego de llevar a cabo una serie de acciones para el desarrollo del concepto de macroestructura textual desde el análisis de textos cortos con el fin de fortalecer los niveles de comprensión de lectura, y de aplicar después este concepto de la lingüística textual en la comprensión y resolución de dichos problemas matemáticos-, son varios los aspectos y aportes que se encontraron:

En cuanto a los prerrequisitos para orientar el proceso de análisis sugerido.

- Si se construye con los estudiantes la definición del enunciado del problema matemático como un texto, con sus elementos y características, esto los incentiva a realizar un análisis literal e inferencial.

- Identificar la habilidad que presenta el estudiante para realizar el análisis literal en 
un enunciado o en un texto cotidiano es fundamental, dado que, a mayor habilidad, mayor predisposición para desarrollar un nivel de análisis superior -en este caso, el inferencial con las macrorreglas-.

- El vocabulario o recuperación léxica, la pronominalización y la puntuación son herramientas del análisis literal que con la práctica se convierten en operaciones mecánicas. Sin embargo, en el análisis de enunciados de situaciones o problemas matemáticos, el docente debe estar en constante orientación hacia la transformación del lenguaje cotidiano en lenguaje matemático, dado que hay proposiciones, oraciones y palabras claves que pueden representar una medida, un valor, e incluso un algoritmo dentro de un problema, las cuales el estudiante generalmente pasa por alto en su lectura e interpretación.

- En el análisis de un enunciado de un problema matemático hay que plantear diferentes preguntas en torno a la interpretación del texto en su totalidad y no reducir las preguntas exclusivamente a la comprensión del dato desconocido o incógnita. Vale la pena mencionar que de la calidad y nivel de exigencia de las preguntas y del trabajo de análisis depende el desarrollo de la habilidad.

En cuanto a la reconstrucción de macroestructuras.

- Al centrar el proceso de solución de un problema en el análisis e interpretación del enunciado como un texto, en el manejo de su información se desplaza y, en cierta medida, se modifica la tendencia del estudiante por aplicar un algoritmo relacionado con el tema visto en clase.

- Luego del análisis literal es esencial el trabajo inferencial desde el uso de las operaciones o macrorreglas, dado que esto facilita no solo la interpretación global del enunciado, sino la selección, organización y relación adecuada de la información, de modo tal que la respuesta se encuentra como producto del análisis y no como producto de la aplicación de un procedimiento.

- La macrorregla de supresión aplicada al texto de un problema facilitó no solo la discriminación de los datos relevantes de los irrelevantes, sino además la identificación y omisión de los distractores, de tal forma que el estudiante se centró en lo esencial de dicha situación.

- La generalización que se realizó con los datos o información después de la supresión permitió corroborar que la información seleccionada fue suficiente, pues plantear la situación desde las ideas propias del estudiante facilita la recordación y la determinación clara de cuál es la información que falta o que complementa la situación.

- El estudiante se debe enfrentar a dos etapas de construcción. La primera es la construcción de la macroestructura o idea fundamental de la situación, lo cual determina, en gran medida, la relación que se establece entre los datos o valores. La segunda es la complementación de la primera construcción después de encontrar la solución para verificar si la información satisface o no la idea construida. 
En cuanto al análisis de problemas matemáticos de estructura multiplicativa.

- Se encontró que, tan solo cuando se ha suprimido, generalizado y construido con la información del enunciado, es el momento adecuado para identificar las clases, tipos o categorías de medidas que caracterizan a los problemas de estructura multiplicativa.

- En la misma forma, al identificar correctamente los tipos de medidas, se facilita la ubicación u organización de los valores propios de cada una de ellas, sus correspondencias y relaciones. De ahí que los valores no se reduzcan simplemente a los términos de un algoritmo, sino a elementos fundamentales entre las relaciones de la información dentro de un problema.

- La construcción de una tabla de correspondencia con las clases de medias, sus valores y relaciones facilitaron la identificación del procedimiento a seguir para la búsqueda del dato o información desconocida que satisfacía la situación.

- La identificación y comprensión de los diferentes tipos de relaciones y correspondencias entre los datos de los problemas de estructura multiplicativa facilitó el desarrollo de cierta habilidad en el proceso de análisis sugerido.

\section{Propuesta metodológica.}

Se planteó una secuencia metodológica de talleres como estrategia para el mejoramiento de los niveles de comprensión y resolución de problemas matemáticos desde el concepto de macroestructura textual para estudiantes de grado quinto; esto con el fin de llevar a cabo actividades secuenciales y significativas que contribuyeran a que los estudiantes desarrollaran procesos mentales, para fortalecer y mejorar sus niveles de comprensión de lectura y de resolución de problemas matemáticos propios de grado quinto.

La organización de dicha propuesta se dio en dos ámbitos: el primero a nivel general, ya que se presentó una secuencia de ocho talleres con temáticas que integraban los dos aspectos claves en esta investigación, como lo son la comprensión de lectura y los problemas de estructura multiplicativa; el segundo ámbito se presentó a nivel específico - es decir, al interior de cada taller, dado que cada uno de ellos se planteaba desde un enfoque constructivista con miras a desarrollar un aprendizaje significativo (Ausubel, 1976) - y constaba de etapas comunes y fundamentales para el desarrollo de dichas temáticas etapas como exploración, socialización, orientación, aplicación y evaluación-.

La temática de los talleres inició con la construcción de la definición contemporánea de texto, sus características y elementos. Luego se identificaron algunas herramientas y actividades que contribuyeron al desarrollo de la comprensión literal de textos, seguidas de las operaciones como la puntuación, contextualización y pronominalización, las cuales son claves en el momento de producir inferencias proposicionales. Se pasó después por la supresión o elisión de información, la generalización y la construcción de la macroestructura de un texto. Se continuó con la identificación de datos conocidos y desconocidos en los enunciados de los problemas matemáticos, la organización de relaciones cuaternarias o terciarias con la información de los problemas. Se finalizó con la aplicación de los temas trabajados a nivel textual en cada uno de los enunciados de los problemas de estructura multiplicativa, que son propios del quinto grado de educación primaria de acuerdo con los 
estándares de competencias (MEN, 2006).

Dicha estructura se observa claramente en la siguiente figura:

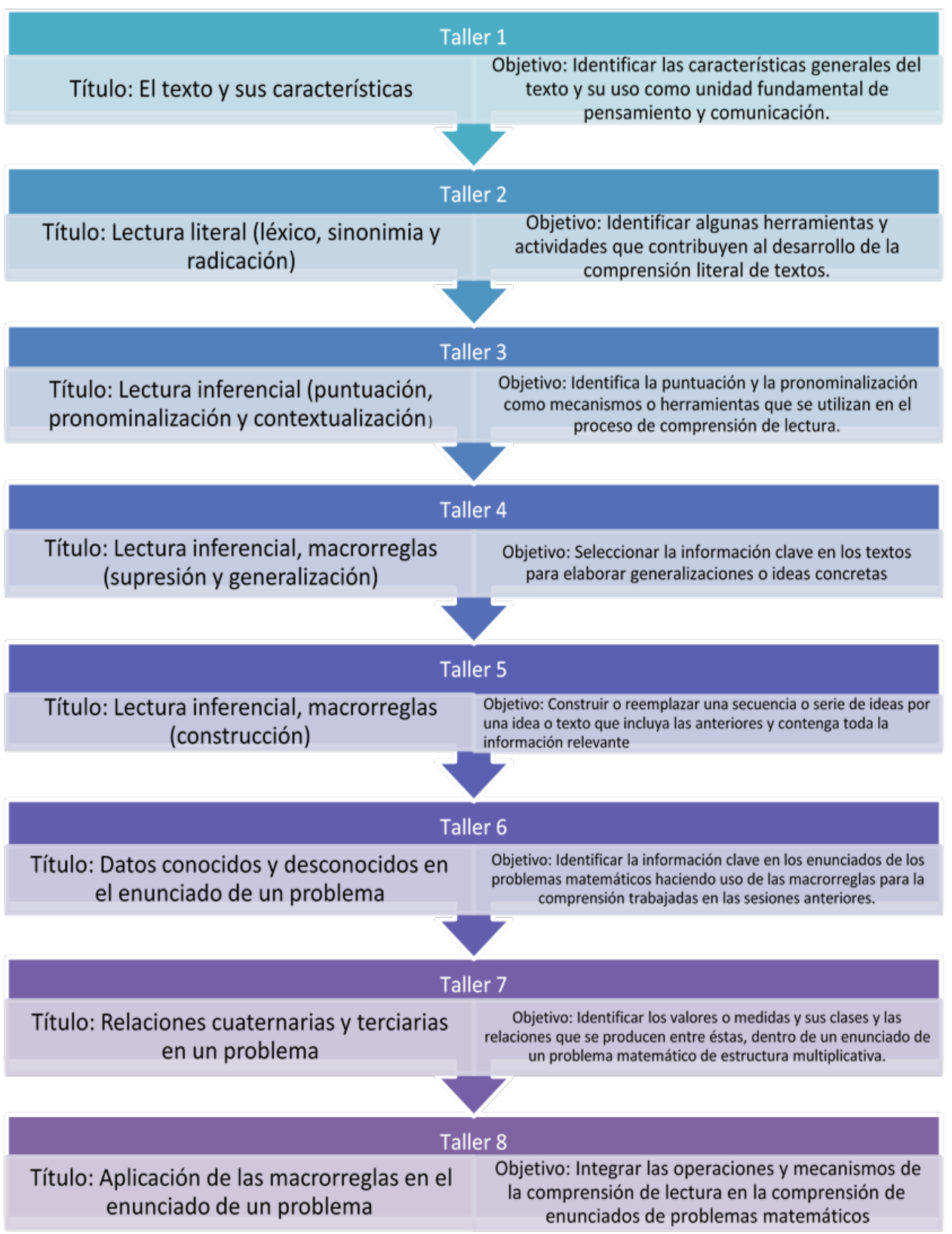

Figura 2. Propuesta metodológica. Fuente: elaboración propia.

\section{Conclusión}

Tanto la resolución de problemas matemáticos de estructura multiplicativa como la comprensión de lectura desde la lingüística del texto son procesos claves en el desarrollo académico de los estudiantes de grado quinto de educación básica. Sin embargo, la investigación mostró que en dichos procesos es donde se presenta mayor dificultad para los estudiantes, por lo cual es necesario y pertinente que el maestro esté en constante búsqueda de metodologías y estrategias didácticas para incentivar el interés del estudiante por el 
desarrollo de procesos mentales que le permitan comprender, analizar y participar en su entorno de manera competente. Por lo tanto, se debe orientar el desarrollo académico del área de matemáticas desde la comprensión de situaciones reales que involucren al estudiante, sus estrategias de solución y procesos mentales, convirtiendo así el análisis y resolución de problemas en ejes centrales de dicha área.

La relación entre comprensión y solución de problemas matemáticos son el eje central. Los problemas matemáticos — trabajados en matemáticas- y la comprensión de textos - trabajados en la asignatura de lengua española - son temas interdependientes. No son procesos aislados, asignados a un campo o disciplina específica, sino que se complementan e integran entre sí, dado que un buen proceso de comprensión facilita entender los enunciados del problema. Su análisis fortalece el proceso mismo de comprensión.

Una propuesta basada en la comprensión de enunciados no solo es pertinente para reducir la distancia entre estas dos áreas, sino que aporta a la superación de dificultades y logra cierto grado de integración entre estas. A la vez se da cumplimiento a las políticas de educación distrital, consignadas en el plan sectorial de Bogotá.

Es claro que no se puede caer en el error de enmarcar a los estudiantes en un método o en una serie de pasos entendidos como un rígido catálogo a seguir, el cual no puede ser modificable a la hora de solucionar problemas. Por el contrario, lo que se propone son estrategias y operaciones sugeridas desde los aportes de Van Dijk (1980) y Zubiría (1995) para el análisis inferencial de los textos. Según Vergnaud (1997), respecto a las características de los problemas de estructura multiplicativa, las dificultades encontradas generalmente radican en la comprensión y análisis de la situación, en la selección y clasificación de la información, en la organización de los datos y en su adecuada correspondencia y relaciones.

Finalmente, uno de los aspectos relevantes a la hora de implementar la propuesta es el diagnóstico detallado del nivel de habilidad del grupo de estudiantes frente a cada una de las categorías de análisis propuestas, de modo que se pueda profundizar en el trabajo de acuerdo con las características del grupo. También es esencial verificar el nivel de desarrollo de algoritmos, pues si los estudiantes no tienen claro el desarrollo de operaciones básicas, esto va a retrasar el proceso. De igual forma, se debe partir del hecho de que es un proceso conjunto para el desarrollo del pensamiento matemático, desde y para el proceso de comprensión lectora, por lo que no se trata de asignar parte del trabajo a un área específica, ni de fraccionar el proceso, sino de convertirlo en un desarrollo continuo, integrado, complementario e interrelacionado.

\section{Referencias}

Arnal, J., Rincón del, D., \& Latorre, A. (1992). Investigación educativa. Metodologías de investigación educativa. Barcelona: Labor.

Ausubel, D. (1976). Psicología educativa: un punto de vista cognoscitivo. México: Editorial Trillas.

Bernárdez, E. (1982). Introducción a la lingüística del texto. Madrid: Espasa.

Blanco, L. (2015). ¿Qué entendemos por problema de matemáticas? En L. Blanco, J. 
Cárdenas, \& A. Caballero, La resolución de problemas matemáticos en la formación inicial de profesores de primaria (pp. 81-91). Extremadura: Universidad de Extremadura.

Blanco, L., \& Caballero A. (2015). Modelo integrado de resolución de problemas. En L. Blanco, J. Cárdenas, \& A. Caballero, La resolución de problemas matemáticos en la formación inicial de profesores de primaria (pp. 109-122). Extremadura: Universidad de Extremadura.

Briz, A., \& Serrano, A. (2018). Aprendizaje de las matemáticas a través del lenguaje de programación R en Educación Secundaria. Educación matemática, 30(1), 133-162. https://dx.doi.org/10.24844/em3001.05

Cárdenas, J., \& Blanco, L. (2015). Resolución de problemas matemáticos como contenido en el currículo de primaria. En L. Blanco, J. Cárdenas, \& A. Caballero (Coords.), La resolución de problemas matemáticos en la formación inicial de profesores de primaria (pp. 23-37). Extremadura, España: Universidad de Extremadura.

Carrillo, J. (1995). La resolución de problemas en matemáticas. Investigación en la escuela, (25), 79-86.

Castro, E. (2017). Abordaje didáctico de la comprensión de los problemas algebraicos en el nivel secundario de la República Dominicana. Transformación, 13(3), 314-326.

Herrada, G., \& Herrada, R. (2017). Análisis del proceso de comprensión lectora de los estudiantes desde el modelo construcción-integración. Perfiles educativos, 39(157), 181197.

House, P., Wallace, M., \& Johnson, M. (1983). Problem Solving as a Focus: How? When? Whose Responsibility? The agenda in action, conference held at the National Council of Teachers of Mathematics (pp. 9-19). Virginia.

Leal, S., \& Bong, S. (2015). La resolución de problemas matemáticos en el contexto de los proyectos de aprendizaje. Revista de Investigación, 39(84), 71-93.

Ministerio de Educación Nacional. (1998). Lineamientos curriculares matemáticas. Bogotá, Colombia: Magisterio.

Montes, A., Rangel, Y., \& Reyes, J. (2014). Comprensión lectora. Noción de lectura y uso de macrorreglas. Ra Ximhai, 10(5), 265-277.

Mora, C. (2003). Estrategias para el aprendizaje y la enseñanza de las matemáticas. Revista de Pedagogía, 24(70), 181-272. Recuperado de http://ve.scielo.org/scielo.php?script=sci_ arttext\&pid=S0798-97922003000200002\&lng=es\&tlng=es.

Murcia, E., \& Henao, J. (2015). Educación matemática en Colombia, una perspectiva evolucionaria. Entre ciencia e ingeniería, 9(18), 23-30.

Ormrod, L. (2005). Transferencia y resolución de problemas. En L. Ormrod (Ed.), Aprendizaje Humano (pp.405-450). Columbus, Ohio: Pearson Education.

Pérez, H. (2006). Comprensión y producción de textos. Bogotá: Magisterio.

Pérez, K., \& Hernández, J. (2014). Aprendizaje y comprensión. Una mirada desde las humanidades. Humanidades Médicas, 14(3), 699-709. 
Pérez, K., Hernández, J., \& Álvarez, M. (2015). Las inferencias en la comprensión de problemas aritméticos en la enseñanza primaria. Varona, (61), 1-10.

Perkins, D. (1995). El contenido: hacia una pedagogía de la comprensión. En D. Perkins (Ed.). La escuela inteligente. Barcelona: Gedisa.

Pino, J. (2013). La resolución de problemas y el dominio afectivo: un estudio con futuros profesores de matemáticas de secundaria. En V. Mellado, L. Blanco, A. Borrachero, \& J. Cárdenas (Coords.), Las emociones en la enseñanza y el aprendizaje de las ciencias y las matemáticas (pp. 117 - 148). Badajoz, España: Grupo Deprofe.

Polya, G. (1965). Cómo plantear y resolver un problema. México, D.F.:Trillas.

Puig, L., \& Cerdán, F. (1995). Problemas aritméticos escolares. Madrid, España: Síntesis.

Rodríguez, M., Gregori, P., Riveros, A., \& Aceituno, D. (2017). Análisis de las estrategias de resolución de problemas en matemática utilizadas por estudiantes talentosos de 12 a 14 años. Educación matemática, 29(2), 159-186. https://dx.doi.org/10.24844/em2902.06

Schoenfeld, A. (1994). Mathematical Thinking and Problem Solving. Hillsdale, NJ: Erlbaum.

Serna, J., \& Díaz, J. (2014). Propuesta didáctica para la comprensión crítica en la Universidad La Gran Colombia. Cuadernos de Lingüística Hispánica, (25), 165-180.

Van Dijk, T. (1980). Estructura y funciones del discurso. Madrid, España: Siglo XXI editores.

Vergnaud, G. (1997). El niño, las matemáticas y la realidad. México: Trillas.

Zubiría de, M. (1995). Teoría de las seis lecturas. Bogotá, Colombia: Famidi, Fundación Alberto Merani para el desarrollo de la inteligencia. 